\title{
A Preliminary Clinical Study of Lesion-Free and Lesion-Active Patients with Oral Lichen Planus and/or Oral Lichenoid Reactions
}

\author{
Foo $\mathrm{SY}^{1}$, Lee $\mathrm{WY}^{1}$, Goh $\mathrm{YC}^{2}$, Siar $\mathrm{CH}^{2 *}$ \\ ${ }_{1}^{1}$ Oral Health Division, Ministry of Health Malaysia, Kuala Lumpur, Malaysia, \\ ${ }^{2}$ Faculty of Dentistry, University of Malaya, Kuala Lumpur, Malaysia.
}

\begin{abstract}
Background: Oral lichen planus (OLP) which belongs to the spectrum of $L P$, is a commonly encountered oral mucosal problem among patients seeking treatment in the Oral Medicine clinic. Its counterpart, oral lichenoid reaction (OLR), which resembled OLP clinically and microscopically, is often encountered in these patients as well. Although the various clinical forms of OLP/OLR are well-recognized, the disease pattern in regards to lesion-active and lesion-free periods in these patients remains poorly characterized. Thus the aim of this study was to determine the clinical and demographic profile of those patients with active OLP/OLR lesions and compare them with those patients who are clinically lesion-free.

Materials and methods: The study subjects comprised 20 patients who attended the Oral Medicine Clinic at the Faculty of Dentistry, University of Malaya for follow-up appointments/management. They were interviewed according to a pre-designed questionnaire, and clinical examination was carried out. All these were conducted under the supervision of Oral Medicine specialists. OLP was diagnosed according to the internationally accepted criteria.

Results: The cohort consisted of predominantly female $(n=14 ; 70 \%)$ and Chinese $(n=9 ; 45 \%)$ patients, with an overall age range of 26-79 years (median, 56 years). Fifteen $(75 \%)$ patients presented with active OLP/OLR lesions and the remaining $5(25 \%)$ were lesion-free. The lesion-active group comprised $3(15 \%)$ males and $12(60 \%)$ females whereas the lesion-free had $3(15 \%)$ males and $2(10 \%)$ females. Reticular LP was the most common type $(n=21 / 35 ; 60 \%)$ and the gingiva was the most prevalent site $(n=21 / 46 ; 45.65 \%)$. Social habits, medical histories and medications were not significantly different between lesion-active and lesion-free OLP patients.

Conclusions: Findings suggest that demographic parameters, lifestyle and systemic diseases do not appear to influence the disease pattern and lesion severity in OLP.
\end{abstract}

Keywords: Clinical features, demographic profile, lesion-free vs lesion-active, oral lichen planus and oral lichenoid reaction

\section{INTRODUCTION}

Oral lichen planus (OLP) is a chronic inflammatory mucocutaneous disorder which occurs in $1-2 \%$ of general population, and is putatively due to an immunologic T cell-mediated process (1-3). Different regions of the world have reported varying prevalence of OLP. Oral lesions in OLP are chronic, and now generally regarded as a potentially malignant disorder (2). Large series based on hundreds of OLP cases from developed countries have described the demographic and clinical characteristics of 
OLP while reports on large series from developing countries remain scarce (4). The information about the demographic and clinical characteristics of OLP from other countries may not be extrapolated to our local population due to differences in ethnic, cultural and demographic profiles. The limited information has led us to conduct a study in this area. Data from this study can be used as local reference in the daily clinical practice and hopefully as a guide to gain some insights into the disease progression of OLP/OLR by examining for clinical disease pattern, symptomology and co-morbities, and to derive a hypothesis to explain the periodic exacerbations and remissions of this chronic disorder.

The main objective of this study was to compare the clinical and demographic characteristics between patients with active OLP/OLR lesions and those who are lesion-free. The other objectives were to study the epidemiological characteristic of OLP/OLR in a selected population in order to acquire data such as prevalence, distribution according to age, gender, ethnicity, habits, oral hygiene, systemic conditions, clinical types and intraoral locations.

OLP occurs most commonly in women older than 40 years of age (3). Mean age is 61 years for women and 58 years for men (4). It commonly affects the mucosa of the oral cavity, but other mucosal sites can be involved namely glans penis, vulvar, vaginal, esophageal, and conjunctiva (5). These lesions typically present bilaterally but may not be symmetrical. They often affect the buccal mucosa, tongue, and gingiva. Gingiva LP or known as desquamative gingivitis, is characterized by an erythematous or ulceration area localized in the attached gingiva associated with small whitish areas. (6) Mucosal lesions persist for many years and usually more chronic compared to skin lesions.

A correct definitive diagnosis of OLP can be made according to its clinical oral manifestation. Nevertheless, oral biopsy with a histopathological evaluation is recommended to confirm the clinical diagnosis (7). Verification of diagnosis between OLR and OLP remains questionable as there are no definite and distinct clinical or histological features that reliably differentiate between them (8). OLR may be considered as a disease by itself or an exacerbation of a pre-existing OLP (6). A recent international consensus proposed the classification of OLR into three main groups which are: 1 . oral lichenoid contact lesions (OLCL), 2. oral lichenoid drug reactions (OLDR) and 3 . oral lichenoid lesions of graft-versus-host disease (9).
OLCL is a delayed immune-mediated hypersensitivity. Clinical features suggestive for OLCL include their proximity to dental restorations (especially amalgam) (10). This type of lesion will resolve within a few months after removal and replacement of the causative material. OLDR may develop after taking medications such as antimalarials, non-steroidal anti-inflammatory drugs (NSAIDS), angiotension converting enzyme inhibitors, diuretics, beta-blockers, oral hypogylcaemics, gold salt, penicillamine, and antiretrovirals. The lesion will resolve after withdrawal of the medications. Lastly, OLL-GVHD is due to acute or chronic GVHD.

The aetiology of the disease process underlying OLP remains unclear, but many causal factors have been implicated. Some of these predisposing factors include anxiety, diabetes mellitus, autoimmune diseases, gastrointestinal diseases, drugs, stress, hypertension, infectious agents(including herpes simplex virus I, herpes virus 6, cytomegalovirus, human papilloma virus, Epstein-Barr virus, Helicobacter pylori and hepatitis virus), dental materials, neoplasms and genetic predisposition (3). A number of previous reports have suggested that patients with OLP have positive correlation with diabetes mellitus and liver disease more often than patients without OLP (11). Conversely, some reports have disputed with these postulations (12). The association between OLP and hepatitis $C$ virus also remains a debatable issue (13). Association of OLP and hypertension remains unclear and controversial. Some authors have reported that there are no association between OLP and cardiovascular diseases. (14) In another study that comprised 5000 Turkish adults, no relation was found between systemic disease and oral mucosal lesion occurrence. Among all examined lesions OLP, leukoplakia were observed in $2.2 \%$ of patients. (15). Another study disclosed that stress is the most frequent cause of acute aggravations of OLP (16).

The oral manifestations of OLP have been classified into six clinical forms namely reticular, plaque-like, papular, atrophic, erosive and bullous. Reticular form is generally asymptomatic while atrophic and erosive lesions result in intense discomfort, burning sensation, difficulties in speaking, eating and swallowing (16). Besides differ in multifocal distribution, plaque lesions consist of similar manifestation to leukoplakia and are more common among tobacco smokers (17).

There is currently no cure for OLP. Management of OLP remains symptomatic. Topical corticosteroid 
(18) remains the treatment of choice though other forms including tacrolimus (19), retinoid (20), photodynamic therapy (21), and cyclosporine have been reported. If lesions are symptomless, treatment is generally not required (3). However, regular review is always advisable for asymptomatic lesions (22).

\section{MATERIALS AND METHODS}

Approval from the Research Ethics Committee [Ethics ID: DF OS1503/0016(U)], Faculty of Dentistry, University of Malaya was obtained prior to the commencement of this elective project.

A total sample of 20 patients seeking specialist treatment for OLP and/or OLR at the Oral Medicine Clinic in the Faculty of Dentistry, University of Malaya from July to August 2015 was included in this study. Inclusion criteria were the diagnosis of OLP lesion with complete clinical and pathology records. For the clinical diagnosis of OLP, the presence of bilateral, mostly symmetrical reticular white striation and/or papules was the clinical inclusion criterion. Exclusion criteria were incomplete records and study cases presenting with oral lesions exhibiting histopathological features of epithelial dysplasia as well as those presenting with drugs and dental restorations implicated in oral lichenoid lesions. All patients were given patient information sheet as reference in this study and were interviewed by two examiners. A pre-designed questionnaire survey form included particulars such as age, gender, ethnicity, occupation, habits, and medical history (patients' underlying systemic diseases, hospitalization, allergies, medication), habits on tobacco, smoking, alcohol taking, betel quid chewing and bruxism were asked. Details on the frequency and duration of these habits were not obtained.

A systematic intra-oral clinical examination was carried out by two examiners under supervision of the Oral Medicine Specialist (CHS) and assisted by her postgraduate trainee (YCG) from the Department of Oral and Maxillofacial Clinical Sciences, Faculty of Dentistry, University of Malaya. Dental light, mouth mirrors, and gauze were used during intra-oral clinical examination.

Biopsies and laboratory tests were carried out by the attending specialist to confirm the diagnosis. OLP or OLR lesions were identified and diagnosed based on the modified WHO diagnostic criteria by van der Meij and van der Waal, 2003 (23). The diagnosis of OLP was based on the following criteria:
- Presence of characteristic bilateral clinical signs of OLP [papular and/ or reticular lesions (Wickham striae) alone or in association with atrophic and erosive lesions]

- Histologic examination of clinical diagnosis through incisional biopsy demonstrating the following microscopic characteristics.

- Presence of a well-defined band-like zone of cellular infiltration that is confined to the superficial part of connective tissues, consisting mainly lymphocytes.

- Signs of "liquefaction degeneration" in the basal cell layer

- Absence of sign of epithelial dysplasia at the moment of first diagnosis

- Absence of suspicion that oral lesions may be related to any drugs or oral restoration.

All patients received information about the outcomes of the clinical assessment, their oral conditions and instructions for oral hygiene maintenance after examination. OLP or OLR lesions were identified and clinical pictures were captured after permission from patients was obtained. The diagnosis with its clinical form of lesion and anatomical location was recorded. The diagnosis of OLP was established according to the clinical features by using six clinical diagnostic criteria in Chamonix, France (24) and of Ingafou et al (10). These are as follow:

- Reticular: keratotic, white striae arranged in a reticular pattern only

- Atrophic: erythematous, with or without keratotic striae

- Erosive: well-defined ulceration with bleeding, with or without white striae

- Plaque-like: white patch, with or without an erythematous area

- Papular: small white papules $(0.5 \mathrm{~mm}$ to $1.0 \mathrm{~mm}$ of diameter) with fine striae in tis periphery

- Bullous: blisters that increase in size and tend to rupture, leaving the surface ulcerated and painful, with fine keratinized striae at the periphery

In patient with more than one clinical type of lesion, such as reticular and erosive, all lesion types were recorded.

\section{STATISTICAL ANALYSIS}

The data collected from each patient were analyzed using descriptive statistics. Chi-square tests were applied to compare the occurrence of OLP and/or 
OLR with ethnicity, gender, medical histories and habits while Mann-Whitney test was used to compare the occurrence of OLP and/or OLR with age. The level of significance was set at $P<0.05$. Data were analyzed with SPSS version 23.0.

\section{RESULTS}

A total of 20 patients participated in this crosssectional study. The results are summarized in Tables 1 to 7 and illustrated in Figures 1-3.

Table 1 shows the distribution of patients with lesion-free and lesion-active of oral lichen planus I oral lichenoid reaction (OLP/OLR) according to gender. There were $6(30.0 \%)$ males and 14 $(70.0 \%)$ females presented with OLP/OLR. Most female patients were having lesion-active OLP/OLR $(60.0 \%)$. Based on Fisher's exact test, differences in gender between patients with lesion-active and lesion-free OLP/OLR were not statistically significant $(P>0.05)$.

Table 1: Distribution of lesion-active and lesion-free OLP/ OLR patients according to gender.

\begin{tabular}{lccccccc}
\hline & \multicolumn{3}{c}{ OLP/OLR patients } & \multicolumn{2}{c}{ Total } \\
\cline { 2 - 6 } Gender & \multicolumn{3}{c}{ Lesion-active } & \multicolumn{2}{c}{ Lesion-free } & & \\
& $(\mathrm{n})$ & $(\%)$ & $(\mathrm{n})$ & $(\%)$ & $(\mathrm{n})$ & $(\%)$ \\
Male & 3 & 15.0 & 3 & 15.0 & 6 & 30.0 \\
Female & 12 & 60.0 & 2 & 10.0 & 14 & 70.0 \\
\hline Total & 15 & 75.0 & 5 & 25.0 & 20 & 100.0 \\
\hline
\end{tabular}

* $P$ value was obtained from Fisher's exact test in Chi-square test. $P=0.131$

Table 2 shows the distribution of lesion-active and lesion-free OLP/OLR patients according to their median age. The ages of the patients from this cross sectional study ranged from 26 to 79 years, with median age of 56 years for both lesion active and lesion free patients. The age of onset in oldest lesion-active patient who received treatment in Oral Medicine clinic, University Malaya was 79 years. For lesion-active OLP/OLR, most patients belonged to median age group of $>56(35 \%)$. Based on MannWhitney test, differences in the ages between lesionactive and lesion-free OLP/OLR patients were not statistically significant $(P>0.05)$.
Table 2: Distribution of lesion-active and lesion-free OLP/ OLR patients according to median age.

\begin{tabular}{|c|c|c|c|c|c|c|}
\hline \multirow{3}{*}{$\begin{array}{l}\text { Median age } \\
(\mathrm{yr})\end{array}$} & \multicolumn{4}{|c|}{ OLP/OLR patients } & \multicolumn{2}{|c|}{ Total } \\
\hline & \multicolumn{2}{|c|}{ Lesion-active } & \multicolumn{2}{|c|}{ Lesion-free } & \multirow[b]{2}{*}{ (n) } & \multirow[b]{2}{*}{$(\%)$} \\
\hline & (n) & $(\%)$ & $(n)$ & $(\%)$ & & \\
\hline$<56$ & 5 & 25.0 & 2 & 10.0 & 7 & 35.0 \\
\hline 56 & 3 & 15.0 & 1 & 5.0 & 4 & 20.0 \\
\hline$>56$ & 7 & 35.0 & 2 & 10.0 & 9 & 45.0 \\
\hline Total & 15 & 75.0 & 5 & 25.0 & 20 & 100.0 \\
\hline
\end{tabular}

* $P$ value obtained from Mann-Whitney test. $P=0.568$.

Mean age: 56.86 years

Table 3 shows the distribution of lesion-active and lesion-free OLP/OLR patients according to their ethnic groups. Chinese showed the highest prevalence in developing lesion-active OLP/OLR which was $8(40.0 \%)$ patients, followed by Malays with $4(20.0 \%)$ patients. In lesion-free OLP/OLR, Indian patients showed the highest prevalence which was $3(15.0 \%)$. The ethnic differences in lesion-active and lesion-free OLP/OLR patients was not statistically significant $(P>0.05)$.

Table 3: Distribution of lesion-active and lesion-free OLP/ OLR patients according to ethnicity.

\begin{tabular}{lcccccc}
\hline Ethnicity & \multicolumn{3}{c}{ OLP/OLR patients } & \multicolumn{2}{c}{ Total } \\
\cline { 2 - 6 } & \multicolumn{2}{c}{ Lesion-active } & \multicolumn{2}{c}{ Lesion-free } & & \\
& $(\mathrm{n})$ & $(\%)$ & $(\mathrm{n})$ & $(\%)$ & $(\mathrm{n})$ & $(\%)$ \\
Malay & 4 & 20.0 & 1 & 5.0 & 5 & 25.0 \\
Chinese & 8 & 40.0 & 1 & 5.0 & 9 & 45.0 \\
Indian & 2 & 10.0 & 3 & 15.0 & 5 & 25.0 \\
Others & 1 & 5.0 & 0 & 0.0 & 1 & 5.0 \\
Total & 15 & 75.0 & 5 & 25.0 & 20 & 100.0 \\
\hline
\end{tabular}

* $P$ value obtained from Fisher's exact test in Chi-square test. $P=$ 0.327

Table 4 shows the distribution of lesion-active and lesion-free OLP/OLR patients according to habits. There were only 3 patients with history of bruxism and 2 patients with alcohol-taking and only 1 patient with smoking habit in lesion-active OLP/ OLR cases. In all lesion-free OLP/OLR patients, no social habits such as alcohol-taking, smoking, betel quid chewing and bruxism were registered. Based on Fisher's exact test, alcohol-taking, smoking, and bruxism were not significantly different between lesion-active and lesion-free OLP/OLR patients $(P>0.05)$. In betel quid chewing, it is a constant since there was no history reported. 
Table 4: Distribution of lesion-active and lesion-free OLP/ OLR patients according to habits.

\begin{tabular}{lccccc}
\hline Social habits & & \multicolumn{5}{c}{ OLP/OLR patients } \\
\cline { 2 - 6 } & & Lesion-active & \multicolumn{2}{c}{ Lesion-free } \\
& Yes & 2 & 10.0 & 0 & 0 \\
Alcohol & No & 13 & 65.0 & 5 & 25.0 \\
$\left({ }^{*} P=1.000\right)$ & Yes & 1 & 5.0 & 0 & 0 \\
Smoking & No & 14 & 70.0 & 5 & 25.0 \\
$\left({ }^{*} P=1.000\right)$ & Yes & 0 & 0 & 0 & 0 \\
Betel quid chewing & No & 15 & 75.0 & 5 & 25.0 \\
('constant) & Yes & 3 & 15.0 & 0 & 0 \\
Bruxism & No & 12 & 60.0 & 5 & 25.0 \\
\hline$\left.{ }^{*} P=0.539\right)$ & &
\end{tabular}

* $P$ value obtained from Fisher exact test in Chi-square test.

Table 5 shows medical histories in lesion-active and lesion-free OLP/OLR patients. Most patients had more than one underlying disease. There were $7(35.0 \%)$ lesion-active and $2(10.0 \%)$ lesionfree OLP/OLR patients presenting with significant medical problems.

Table 5: Distribution of medical history in lesion-active and lesion-free OLP/OLR patients.

\begin{tabular}{|c|c|c|c|c|c|c|}
\hline \multirow[t]{3}{*}{ Medical history } & \multicolumn{4}{|c|}{ OLP/OLR patients } & \multicolumn{2}{|c|}{ Total } \\
\hline & \multicolumn{2}{|c|}{ Lesion-active } & \multicolumn{2}{|c|}{ Lesion-free } & \multirow[b]{2}{*}{ (n) } & \multirow[b]{2}{*}{$(\%)$} \\
\hline & (n) & $(\%)$ & (n) & $(\%)$ & & \\
\hline No disease & 3 & 15.0 & 1 & 5.0 & 4 & 20.0 \\
\hline Single disease & 5 & 25.0 & 2 & 10.0 & 7 & 35.0 \\
\hline $\begin{array}{l}\text { More than one } \\
\text { diseases }\end{array}$ & 7 & 35.0 & 2 & 10.0 & 9 & 45.0 \\
\hline Total & 15 & 75.0 & 5 & 25.0 & 20 & 100.0 \\
\hline
\end{tabular}

${ }^{*} P$ value from Fisher's exact test in Chi-square test. $P=1.000$

Table 6 shows the distribution of OLP/OLR patients according to the underlying medical histories. Various systemic diseases, including hypertension, diabetes mellitus, allergies among lesion-active and lesion-free OLP/OLR patients were not statistically significant difference $(P>0.05)$ according to Fisher's exact test in Chi-square test. Hypertension, hypercholesterolaemia and allergies were the three most common medical diseases recorded in our series which were $(14.71 \%)$ each. followed by diabetes mellitus $(11.76 \%)$. Others namely endocrine, infectious disease, cancer, asthma, gastrointestinal disease, COPD were rare and categorized as miscellaneous group of medical problems encountered.

Table 6: Prevalence of OLP/OLR according to the underlying systemic diseases

\begin{tabular}{lccc}
\hline Systemic diseases & $\mathrm{n}$ & $\%$ & $P$ value \\
\hline Hypertension & 5 & 14.71 & 1.000 \\
\hline Diabetic mellitus & 4 & 11.76 & 0.530 \\
\hline Hypercholesterolaemia & 5 & 14.71 & 0.266 \\
\hline Allergy & 5 & 14.71 & 0.249 \\
\hline Asthma & 3 & 8.82 & 1.000 \\
\hline Endocrine & 3 & 8.82 & 0.539 \\
\hline Cancer & 3 & 8.82 & 0.539 \\
\hline Arthritis & 2 & 5.88 & 0.447 \\
\hline Infectious diseases & 1 & 2.94 & 1.000 \\
\hline Gastrointestinal diseases & 1 & 2.94 & 0.250 \\
\hline COPD* & 1 & 2.94 & 1.000 \\
\hline Cardiovascular disease & 1 & 2.94 & 1.000 \\
\hline Total & 34 & 100.0 & \\
\hline
\end{tabular}

*COPD, chronic obstructive pulmonary disease

Table 7 shows the medication taken by lesion-active and lesion-free OLP/OLR patients. Antihypertensive drugs $(25.0 \%)$ and antidiabetic drugs $(20.0 \%)$ were the two most common prescriptions recorded. However, antihypertensive drug, antidiabetic drug and others showed no statistically significant differences according to Fisher's exact test.

Table 7: Medication taken by lesion-active and lesion-free OLP/OLR patients.

\begin{tabular}{lccccc}
\hline Medications taken & \multicolumn{5}{c}{ OLP/OLR patients } \\
\cline { 2 - 6 } & \multicolumn{5}{c}{$\begin{array}{c}\text { Lesion- } \\
\text { active }\end{array}$} \\
& & Lesion-free & $(\%)$ & $(\mathrm{n})$ & $(\%)$ \\
Antihypertensives & Yes & 5 & $25.0 \%$ & 0 & $0 \%$ \\
$\left({ }^{*} P=0.266\right)$ & No & 10 & $50.0 \%$ & 5 & $25.0 \%$ \\
Antihypercholesterolaemia & Yes & 5 & $25.0 \%$ & 0 & $0 \%$ \\
$\left({ }^{*} P=0.266\right)$ & No & 10 & $50.0 \%$ & 5 & $25.0 \%$ \\
Antidiabetics & Yes & 4 & $20.0 \%$ & 0 & $0 \%$ \\
$\left({ }^{*} P=0.530\right)$ & No & 11 & $55.0 \%$ & 5 & $25.0 \%$ \\
Miscellaneous & Yes & 8 & $40.0 \%$ & 2 & $0 \%$ \\
$\left({ }^{*} P=1.000\right)$ & No & 7 & $35.0 \%$ & 3 & $25.0 \%$ \\
\hline
\end{tabular}

${ }^{*} P$ value obtained from Fisher's exact test in Chi-square test. 
Figure 1 shows the distribution of affected sites in lesion-active OLP/OLR patients. Gingiva was the most common site of involvement in each form $(45.65 \%)$, followed by buccal mucosa $(33.33 \%)$, vestibule $(13.04 \%)$, palate $(4.76 \%)$ and lateral tongue $(4.76 \%)$.

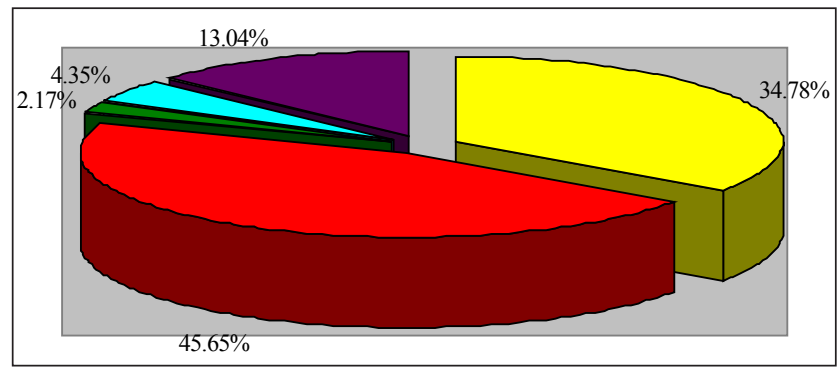

$\square$ Buccal mucosa $\square$ Gingiva $\square$ Palate $\square$ Lateral tongue $\square$ Vestibule

Figure 1: Distribution of affected sites in lesion-active OLP/ OLR patients

Figure 2 shows the distribution of type of lesion in lesion-active OLP/OLR patients. Reticular form $(61.76 \%)$ was the most common type of lesions, followed by atrophic (32.35\%), erosive (2.94\%) and plaque $(2.94 \%)$. Only 1 patient presented with desquamative gingivitis.

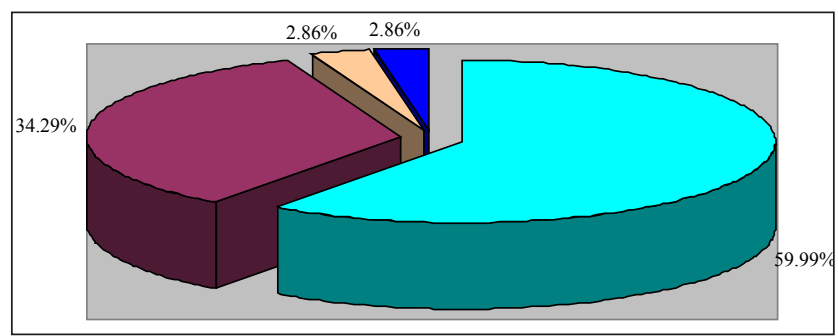

$\square$ Reticular $\square$ Atrophic $\square$ Erosive $\square$ Plaque

Figure 2: Distribution of type of OLP/OLR in lesion-active patients

Figure 3 A-D shows examples of lesion-active patients presenting with common forms of OLP/OLR.
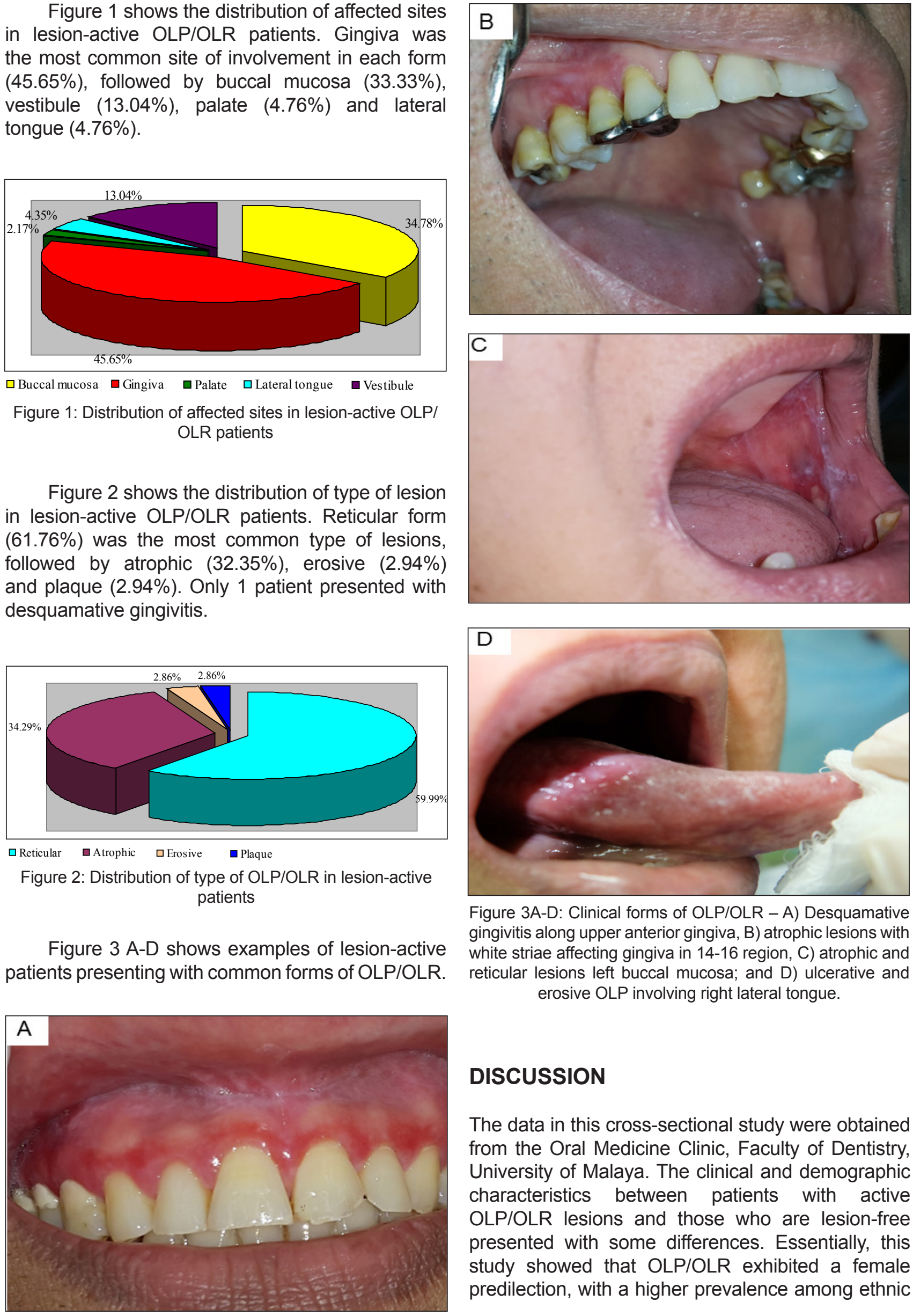

Figure 3A-D: Clinical forms of OLP/OLR - A) Desquamative gingivitis along upper anterior gingiva, $B$ ) atrophic lesions with white striae affecting gingiva in 14-16 region, C) atrophic and reticular lesions left buccal mucosa; and D) ulcerative and erosive OLP involving right lateral tongue.

\section{DISCUSSION}

The data in this cross-sectional study were obtained from the Oral Medicine Clinic, Faculty of Dentistry, University of Malaya. The clinical and demographic characteristics between patients with active OLP/OLR lesions and those who are lesion-free presented with some differences. Essentially, this study showed that OLP/OLR exhibited a female predilection, with a higher prevalence among ethnic 
Chinese, preferentially affected the gingiva and buccal mucosa, and presented mostly in the reticular form.

Demographic analysis confirmed that OLP/ OLR among Malaysian patients exhibits a female predilection although the prevalence rate differed from other studies. We found that the ratio of female to male in lesion-active OLP/OLR was $4: 1$ which is in similar to the study among Thai patients conducted by Thongprasom et al. in 2010 (25). However, there was a lower female to male ratio reported in China (1.9:1) by Xue et al. in 2005(14) and in UK (1.75:1) by Ingafou et al. (10) .

Our demographic results also confirmed that OLP/OLR affects late adult life. The mean age for our study was 56.85 years old in both lesion-free and lesion-active patients. However, according to studies from China (14) and UK (10), the mean ages were 50.4 years and 52.0 years respectively which were slightly lower compared to the mean age of our study. Studies from Spain (26) and Italy (27) obtained mean age of occurrence of OLP/OLR at 56.4 and 56.7 years which were similar to our study.

Due to variations in the ethnic population composition from one country to the next, we did not compare our results on ethnic prevalence rates with those on OLP/OLR from other countries. In our study, we found that a high percentage of Chinese (45\%) presented with OLP/OLR. This finding differed from Yaacob et al.'s series (28) which reported a higher percentage of Indian (45.5\%) with OLP/OLR. It is noteworthy that the percentage of Chinese with OLP/OLR (41.6\%) in Yaacob et al.'s study was slightly lower than in our study. A plausible explanation for this dissimilarity in findings could be due to differences in the selection criteria adopted.

No distinct predominant social habit among OLP/OLR patients was identified in this study. We found that more than $90 \%$ of the patients did not have habits of alcohol-taking and smoking. These observations compared favorably with previous studies. Eisen et al. reported that most OLP patients in their study did not show higher prevalence on cigarette smoking or alcohol consumption (16). Likewise, Carbone et al showed that $77.8 \%$ of the OLP patients are non-smokers and $87.7 \%$ nondrinkers (4).

Our analysis on the prevalence of systemic diseases and medications among OLP/OLR patients indicated that the predominant diseases recorded were hypertension, hypercholesterolaemia and allergies which were present in $14.71 \%$ each, followed by diabetes mellitus (11.76\%). These results differed from Croatian report (29) where hypercholesterolaemia and allergies also share the same percentage with hypertension. In line with these disease prevalences, antihypertensive and anti-hypercholesterolaemia drugs were the two most frequent types of medication used followed by hypoglycaemic drugs.

In our analysis on site distribution in lesion-active OLP/OLR patient, our results contradicted those of Eisen et al.'s study (7). Majority of our patients had lesions on more than one site, most commonly on the gingiva, followed by buccal mucosa, vestibule, lateral tongue and palate. Lesions on floor of mouth, labial mucosa were uncommon. A recent comparative study between Thai and Croatian patients reported that the most common site was buccal mucosa, followed by tongue, gingiva and palate (29). The study of 674 OLP patients in China demonstrated that the other common sites were tongue, lip and gingiva (14), while the study of 690 OLP patients in USA found that the other common sites of involvement were tongue, gingiva and lower lip (16). These different findings could be described to geographic differences and racial make-up, as well as the size of the current sample.

As for the type of OLP lesion, reticular form dominated in this study (60.0\%) and this was in accordance with the studies in China, Japan, Spain, Croatia and UK. (10, 14, 29-31).

\section{CONCLUSIONS}

In summary, the present study showed that OLP/ OLR occurred more frequently in Chinese women. The highest prevalence for women was found in median age group >56 years. Reticular lichen planus was the most common type while the most frequently involved site was gingiva, followed by buccal mucosa, vestibule, lateral tongue and palate. Findings suggest that demographic parameters, lifestyle and systemic diseases do not appear to influence the disease pattern and lesion severity in OLP.

\section{LIMITATION}

The main setback encountered during this study is small sample size which may have led to the results being statistically not significant. Therefore it is 
impossible to establish robust data on prevalence and incidence of OLP/OLR among Malaysians based on a small number of patients who have been referred to the Oral Medicine Clinic of the Faculty of Dentistry, University of Malaya.

\section{ACKNOWLEDGEMENTS}

Grateful thanks to the following for their valuable contributions: Master of Clinical Dentistry in Oral Medicine and Oral Pathology trainees (Drs. Nurmawarnis Mat Hussin, Sarvambika Kazakydasan, Salina Din and Zarina Abdul Karim) for their help in clinical photography and biopsy-taking, Ms. Najihah binti Lokman for her expert statistical help, and to the Oral Medicine Clinic nurses for their assistance and support. This study is partly funded by FP0322015A.

\section{DECLARATION OF INTEREST}

The authors report no conflicts of interest. The authors alone are responsible for the content and writing of the paper.

\section{REFERENCES}

1. Axéll $T$, Rundquist L. Oral lichen planus-a demographic study. Community Dentistry and Oral Epidemiology. 1987; 15(1): 52-6.

2. Van Dis $M$, van der Meij E, Schepman K, Smeele L, van der Wal J, Bezemer P, et al. A review of the recent literature regarding malignant transformation of oral lichen planus. Oral Surgery, Oral Medicine, Oral Pathology, Oral Radiology, and Endodontology. 1999; 88(3): 307-10.

3. Scully C, Beyli M, Ferreiro MC, Ficarra G, Gill Y, Griffiths M, et al. Update on oral lichen planus: etiopathogenesis and management. Critical Reviews in Oral Biology \& Medicine. 1998; 9(1): 86-122.

4. Carbone M, Arduino P, Carrozzo M, Gandolfo S, Argiolas M, Bertolusso G, et al. Course of oral lichen planus: a retrospective study of 808 northern Italian patients. Oral Diseases. 2009; 15(3): 235-43.

5. Setterfield JF, Neill S, Shirlaw PJ, Theron J, Vaughan R, Escudier M, et al. The vulvovaginal gingival syndrome: a severe subgroup of lichen planus with characteristic clinical features and a novel association with the class II HLA DQB1 0201 allele. Journal of the American Academy of Dermatology. 2006; 55(1): 98-113.

6. Ismail SB, Kumar SK, Zain RB. Oral lichen planus and lichenoid reactions: etiopathogenesis, diagnosis, management and malignant transformation. Journal of Oral Science. 2007; 49(2): 89-106.

7. Eisen D, Carrozzo M, Bagan Sebastian JV, Thongprasom K. Number V Oral lichen planus: clinical features and management. Oral Diseases. 2005; 11(6): 338-49.

8. McCartan B, McCreary C, Healy C. Studies of drug-induced lichenoid reactions: criteria for case selection. Oral Diseases. 2003; 9(4): 1634.

9. Al-Hashimi I, Schifter M, Lockhart PB, Wray $D$, Brennan M, Migliorati CA, et al. Oral lichen planus and oral lichenoid lesions: diagnostic and therapeutic considerations. Oral Surgery, Oral Medicine, Oral Pathology, Oral Radiology, and Endodontology. 2007; 103: S25. e1-S. e12.

10. Ingafou M, Leao J, Porter S, Scully C. Oral lichen planus: a retrospective study of 690 British patients. Oral Diseases. 2006; 12(5): 463-8.

11. Lowe N, Cudworth A, Clough S, Bullen M. Carbohydrate metabolism in lichen planus. British Journal of Dermatology. 1976; 95(1): 9-12.

12. Machado A, Sugaya N, Migliari D, Matthews R. Oral lichen planus. Clinical aspects and management in fifty-two Brazilian patients. The West Indian Medical Journal. 2004; 53(2): 1137.

13. Tanei R, Watanabe K, Nishiyama S. Clinical and histopathologic analysis of the relationship between lichen planus and chronic hepatitis $\mathrm{C}$. The Journal of Dermatology. 1995; 22(5): 31623.

14. Xue JL, Fan MW, Wang SZ, Chen XM, Li Y, Wang L. A clinical study of 674 patients with oral lichen planus in China. Journal of Oral Pathology \& Medicine. 2005; 34(8): 467-72.

15. Cebeci A, Gülşahi A, Kamburoglu K, Orhan B-K, Oztaş B. Prevalence and distribution of oral mucosal lesions in an adult Turkish population. Med Oral Patol Oral Cir Bucal. 2009; 14(6): E272-7.

16. Eisen D. The clinical features, malignant potential, and systemic associations of oral lichen planus: a study of 723 patients. Journal of the American Academy of Dermatology. 2002; 46(2): 207-14. 
17. Thorn J, Holmstrup P, Rindum J, Pindborg J. Course of various clinical forms of oral lichen planus. A prospective follow up study of 611 patients. Journal of Oral Pathology \& Medicine. 1988; 17(5): 213-8.

18. Carrozzo M, Gandolfo $S$. The management of oral lichen planus. Oral Diseases. 1999; 5(3): 196-205.

19. Byrd JA, Davis MD, Bruce AJ, Drage LA, Rogers RS. Response of oral lichen planus to topical tacrolimus in 37 patients. Archives of Dermatology. 2004; 140(12): 1508-12.

20. Branchet M, Boisnic S, Pascal F, Ben SL, Rostin $M$, Szpirglas $H$, editors. [Topical tretinoin in the treatment of lichen planus and leukoplakia of the oral mucosa. A biochemical evaluation of the keratinization]. Annales de Dermatologie et de Venereologie; 1993.

21. Sadaksharam J, Nayaki K, Panneer Selvam N. Treatment of oral lichen planus with methylene blue mediated photodynamic therapy-a clinical study. Photodermatology, Photoimmunology \& Photomedicine. 2012; 28(2): 97-101.

22. Krutchkoff DJ, Cutler L, Laskowski S. Oral lichen planus. Journal of Oral Pathology \& Medicine. 1978; 7(1): 1-7.

23. Van der Meij E, Van der Waal I. Lack of clinicopathologic correlation in the diagnosis of oral lichen planus based on the presently available diagnostic criteria and suggestions for modifications. Journal of Oral Pathology \& Medicine. 2003; 32(9): 507-12.

24. Lodi G, Scully C, Carrozzo M, Griffiths M, Sugerman PB, Thongprasom K. Current controversies in oral lichen planus: report of an international consensus meeting. Part 2. Clinical management and malignant transformation. Oral Surgery, Oral Medicine, Oral Pathology, Oral Radiology, and Endodontology. 2005; 100(2): 164-78.

25. Thongprasom K, Youngnak Piboonratanakit $\mathrm{P}$, Pongsiriwet $\mathrm{S}$, Laothumthut $\mathrm{T}$, Kanjanabud P, Rutchakitprakarn L. A multicenter study of oral lichen planus in Thai patients. Journal of Investigative and Clinical Dentistry. 2010; 1(1): 29-36.

26. Bermejo Fenoll A, Sánchez Siles M, López Jornet P, Camacho Alonso F, Salazar Sánchez $\mathrm{N}$. A retrospective clinicopathological study of 550 patients with oral lichen planus in south eastern Spain. Journal of Oral Pathology \& Medicine. 2010; 39(6): 491-6.

27. Gandolfo S, Richiardi L, Carrozzo M, Broccoletti $\mathrm{R}$, Carbone M, Pagano M, et al. Risk of oral squamous cell carcinoma in 402 patients with oral lichen planus: a follow-up study in an Italian population. Oral Oncology. 2004; 40(1): 77-83.

28. Yaacob HB, Tan PL, Ngeow WC. Malignancy in oral lichen planus: a review of a group from the Malaysian population. Journal of Oral Science. 2002; 44(2): 65-71.

29. Thongprasom K. Oral lichen planus: a retrospective comparative study between Thai and Croatian patients. Acta Dermatovenerologica Croatica. 2009; 17(1): 2-8.

30. Bagan-Sebastian J, Milian-Masanet $M$, Penarrocha-Diago M, Jimenez Y. A clinical study of 205 patients with oral lichen planus. Journal of Oral and Maxillofacial surgery. 1992; 50(2): 116-8.

31. Nagao $T$, Ikeda $N$, Fukano $H$, Hashimoto $S$, Shimozato K, Warnakulasuriya S. Incidence rates for oral leukoplakia and lichen planus in a Japanese population. Journal of Oral Pathology \& Medicine. 2005; 34(9): 532-9.

\section{Corresponding author:}

\section{Chong Huat Siar}

Department of Oral and Maxillofacial Surgical \& Medical Sciences,

Faculty of Dentistry, University of Malaya, 50603 Kuala Lumpur, Malaysia.

E-mail Address: chsiar@gmail.com 